\title{
The public health scientist as informal logician
}

\author{
Louise Cummings
}

Published online: 6 December 2011

(c) Swiss School of Public Health 2011

Few fields have been as receptive to ideas from other disciplines as public health. Sociological concepts and theories have helped to shape our understanding of the pervasive influence of class, race and gender on the health of populations. In the same way, I want to argue in this commentary that a branch of logical study known as informal logic can bring new insights to public health.

Informal logic is the study of those forms of reasoning which are not amenable to analysis within formal (deductive) logic. This discipline emerged as a result of dissatisfaction with the privileging or idealising of deductive reasoning in logic, a tendency known as 'deductivism' (Johnson 2011). Whilst informal logicians acknowledge that people can and do engage in deductive reasoning, they argue that in everyday affairs they are much more likely to employ plausible or presumptive reasoning. Although this type of reasoning does not conform to deductive ideals of validity and soundness, it is particularly well adapted to epistemic features of the contexts in which arguments are advanced. These features include the lack of knowledge and uncertainty that attend many public health problems.

The emergence of a distinct discipline of informal logic resonates with developments in public health. In their introduction of a novel synthetic epidemic paradigm, Christakos et al. (2005, p 22) acknowledge a role for modes of reasoning 'that may not be found in formal logic'. These modes or styles of reasoning are manifest in, and adapted to, the uncertainty which pervades public health: 'one can hardly overestimate the importance of the argumentation modes and styles of reasoning under conditions of

L. Cummings $(\varangle)$

School of Arts and Humanities, Nottingham Trent University, Clifton Campus, Clifton Lane, Nottingham NG11 8NS, UK

e-mail: louise.cummings@ntu.ac.uk uncertainty, for they form an essential part of the background intellectual context of public health inquiry' (Christakos et al. 2005, p 55). An emphasis on reasoning strategies in the management of uncertainty is now evident in public health (Plant 2008; Vaughan and Tinker 2009).

In Cummings (2002, 2009, 2010, 2011), I examined a group of reasoning strategies used by scientists who were called upon to make assessments of the public health risks posed by the emergence of bovine spongiform encephalopathy (BSE) in British cattle. These strategies, which included arguments from ignorance and analogical arguments, were commonly found in the reasoning of scientists who sat on expert scientific committees such as the Spongiform Encephalopathy Advisory Committee (SEAC). Although these arguments have traditionally been characterised as 'fallacies' (i.e. weak forms of reasoning), it was demonstrated in these studies that they enabled scientists to bridge 'gaps' in knowledge. To this extent, they were not so much logical flaws in reasoning as facilitative strategies that allowed scientists to form judgements about complex problems in the absence of knowledge. Two uses of these strategies during BSE risk assessments are shown below:

(A) The pathogenesis of BSE is similar to the pathogenesis of scrapie (major premise).

The distal ileum is not infective in lambs (minor premise).

The distal ileum will not be infective in calves (conclusion).

(B) There is no evidence of BSE transmission via the optic nerve in cattle (premise).

The optic nerve in cattle does not transmit BSE (conclusion). 
Of relevance to an assessment of the rationality of these arguments are the findings of a BSE pathogenesis experiment which was conducted at the Central Veterinary Laboratory in Weybridge, Surrey. The results of this experiment were first known in June 1994. This was after the time when scientists were trying to establish which bovine tissues to include in the human Specified Bovine Offal ban (argument (A) above), but before the time when SEAC scientists were assessing the BSE health risks of bovine optic nerve (argument (B) above). With this timescale in mind, it is apparent that both arguments had certain rational merits for the scientists who used them. By drawing a similarity between BSE and scrapie (a transmissible spongiform encephalopathy of sheep), the analogical argument in (A) found scientists using welldeveloped knowledge of the pathogenesis of scrapie to circumvent their lack of knowledge of the pathogenesis of BSE. To the extent that (A) was advanced before results of the BSE pathogenesis experiment were known, and at a time when epidemiological, histological and molecular evidence suggested that BSE and scrapie were related diseases, it is clear that this analogical argument was rationally warranted. Arguments from ignorance are rationally warranted when a knowledge base is complete or closed and has been exhaustively searched. Upon completion of the BSE pathogenesis experiment, it is clear that just such a knowledge base was available to SEAC scientists who advanced the argument from ignorance in (B) these scientists had acquired a complete knowledge base about the pathogenesis of BSE and had exhaustively searched the contents of that base.
It can be seen that arguments (A) and (B) served to bridge 'gaps' in scientists' knowledge of BSE through different but equally effective means (an analogical template and a closed, exhaustively searched knowledge base, respectively). It is to this extent that I recommend these arguments, and informal logic generally, as a valuable area of enquiry for public health scientists who must confront issues of uncertainty in reasoning.

\section{References}

Christakos G, Olea RA, Serre ML, Yu H-L, Wang L-L (2005) Interdisciplinary public health reasoning and epidemic modelling: the case of black death. Springer, The Netherlands

Cummings L (2002) Reasoning under uncertainty: the role of two informal fallacies in an emerging scientific inquiry. Informal Logic 22:113-136

Cummings L (2009) Emerging infectious diseases: coping with uncertainty. Argumentation 23:171-188

Cummings L (2010) Rethinking the BSE crisis: a study of scientific reasoning under uncertainty. Springer, Dordrecht

Cummings L (2011) Considering risk assessment up close: the case of bovine spongiform encephalopathy. Health Risk Soc 13:255-275

Johnson RH (2011) Informal logic and deductivism. Stud Logic 4:17-37

Plant A (2008) When action can't wait: investigating infectious disease outbreaks. In: Bammer G, Smithson M (eds) Uncertainty and risk: multidisciplinary perspectives. Earthscan, London, pp 45-54

Vaughan E, Tinker T (2009) Effective health risk communication about pandemic influenza for vulnerable populations. Am J Public Health 99:S324-S332 